\title{
NURSE LED WARD ROUNDS - A VALUABLE CONTRIBUTION TO NEUROLOGICAL PATIENTS
}

\author{
Renuka.K Hemapriya.S, Anbu.M*
}

\begin{abstract}
Nurses are integral part of the multidisciplinary team, providing care from planning, implementing and evaluating patient's condition. Neurological patients which includes stroke, head injury, paralyzed patients, post operative craniotomy patients, seizure and coma patients are totally dependent on the care giver.. Therefore it is imperative for the nurses to have keen rounds and care for these patients towards recovery. The nurse led ward rounds delineates three phases which depicts the activities that, a nurse should carry out before, during and after nurse led ward rounds. The nurse takes up multiple roles such as Advocate, Chaperone, Transitor, Informator, Organizer and Nurse centered. Although it is important to have interprofessional staff collaboration for successful patient management, nurse-physician collaboration during rounds occur infrequently. To improve the way nurses manage neurological patients in an acute setting, a nurse-led ward round helps to ensure patient care and safety.
\end{abstract}

\section{Key words: Nurse led Ward Rounds, Neurological Patients, Communication, Advocacy}

\section{Introduction:}

Ward round has been a historical clinical method of inter-professional collaboration to support inpatient care by exchanging information and discussing plan of care, treatment goals, and discharge plans for the patient ${ }^{2}$.Although traditional ward rounds offer the opportunity for doctors to spend quality time with patients, reports indicate the experience to be brief for patients, with little opportunity to ask questions, etc. For example, nurses generally spend more time at the bedside of patients and their families, and therefore observe behaviors that doctors do not see at first hand during their brief rounds. ${ }^{17}$

Therefore, we believe that improvements in the conduct of ward rounds may lead to better patientcentered care. Hence, the inclusion of nurses in ward rounds can align professional priorities and facilitate a shared understanding of the patient's needs ${ }^{18}$.

Nurses have a crucial role on ward rounds, not only sharing key information between the patient and the healthcare team, but also supporting patients in articulating their views and preferences. Absence of a nurse at the bedside has clear consequences for communications, ward-round efficiency and patient safety ${ }^{9}$.

\section{Scope of Nurse Led Ward Rounds:}

- Nurses have a vital role in ward rounds and should make it a priority to attend.

- Nurse led Ward rounds provide a link between patients' admission to hospital and their discharge or transfer elsewhere.

- Ensure the continuity of care provided to the patients.

- It eliminates many misconceptions between physician and the nurse.

- It enables the nurse to rectify the doubts of the patients and give them assurance.

- It ensures the involvement of patient and care takers in clinical decisions.

- It helps the new comers in the ward such as the nursing students to know about the patients and their conditions ${ }^{4}$

* Prof. Dr. Renuka.K, Principal. Ms.Hemapriya.S, Lecturer. Ms. Anbu.M, Lecturer Dept. of Medical Surgical Nursing, KGNC, Puducherry 607402, India 


\section{Table 1. Nurse Led Ward Rounds Proforma}

\section{ESSENTIAL STROKE CARE}

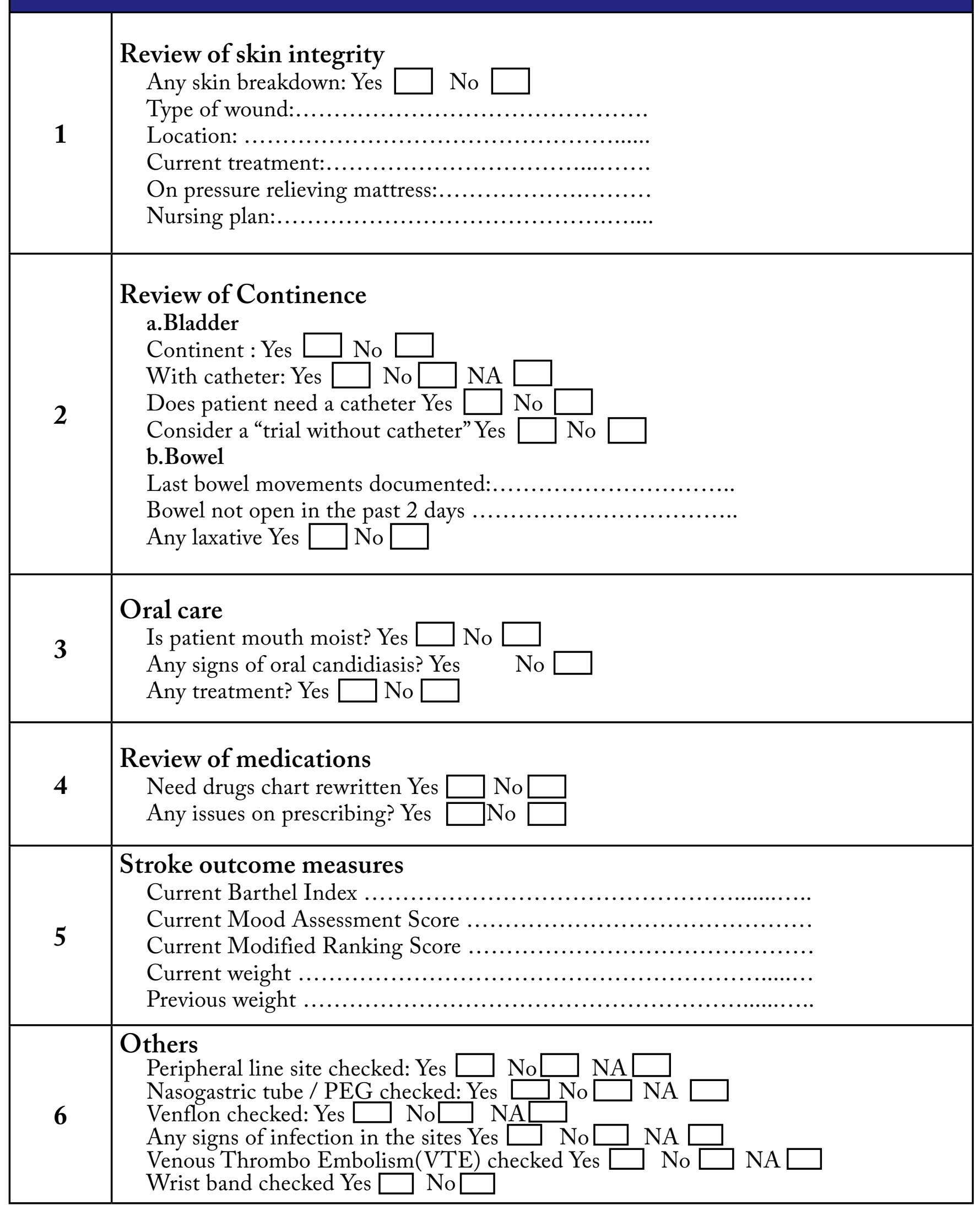




\section{Process of nurse led ward rounds in neurological units.}

The nurse led team consists of clinical nurse specialist, nurse manager, ward supervisor and staff nurse. The rounds will be carried out every Monday of the week. The time spend with each patient varies according to the condition of the patient. It usually ranges from ten to fifteen minutes per patient ${ }^{21}$. The rounds provide an opportunity for the multidisciplinary team to listen to each patient's narrative and jointly interpret the concerns. From this the nurse unfolds diagnosis, management plans, prognosis formation and the opportunity to explore social, psychological, rehabilitation and placement issues ${ }^{20}$. During the rounds the team addresses patient needs and makes appropriate nursing diagnosis and goals. Elma $\mathrm{J}$ and Julia $\mathrm{S}$ suggested the use of a proforma for patient assessment in nurse led ward rounds among neurological patient ${ }^{14}$ (Table 1). After each patients rounds the nursing team places the completed proforma in the medical notes ${ }^{6}$.

Three distinct stages to nurse led ward rounds, each of which has equal importance.

\section{Antecedents (Before)}

Key activities before a nurse led ward round are

- Orienting to patient profile, demographic data and history of the patient.

- Establishing results of investigations such as blood and electrolytes, coagulation profile, Renal Profile, CSF analysis report, CT \& MRI, $\mathrm{X}$ ray, ECG, EEG.

- If the patient is conscious, the patients will be informed that nurse led ward rounds will be taking place, in case of unconscious patients it will be informed to the patient care giver to address the patient's problems and needs ${ }^{5}$.

\section{Critical attributes (During)}

This refers to the key activities, that is integral to nurse led ward rounds

- Obtain a complete neurological and pertinent general history from an adult taking into account age and mental state.
- Distinguish between signs and symptoms that are physiological and those that are the result of a psychological disorder ${ }^{22}$.

- Review of patient's medication management. E.g. culture and sensitivity report in case of antibiotics, therapeutic levels of medications, managing the adverse affects of medications administered and inspects the drug chart to see if the prescribed medications is administered following eight rights ${ }^{22}$.

- The nursing team inspects major pressure areas, start bladder strategies, ensuring pressure relieving bed mattress, ensuring patient's bowel movements, inspecting for any oral thrush etc ${ }^{23}$.

- The nurse also inspects the neurological patient's outcome measures on a weekly basis to evaluate whether neurological patients are improving through provision of rehabilitation therapy, nursing care and treatment ${ }^{23}$.

- The team also inspects the peripheral line/NG tube/ percutaneous gastrostomy tube for any signs of infection around the insertion site and ensures the need for change.

- Decision making and documentation of care. E.g. oral care, back care, perineal care, skin care.

- Outline a plan of investigation including laboratory tests, neuro imaging, and other investigations. Judgment should be exercised taking into account the patient's age, general health risk, cost of investigations, and epidemiology of the disease.

- Formulate an appropriate nursing diagnosis with respect to common signs or symptoms involving the nervous system.

- Review of patients going home (pre-discharge); Pre-discharge occupational therapy home assessments, falls-prevention strategies and functional independence in neurological patients ${ }^{4}$.

\section{Consequences (After)}

Once the ward round is over, a number of activities will be necessary:

- The progress of the patient will be discussed.

- The senior nurse will be presenting the findings of nurse led ward rounds in weekly multidisciplinary meeting.

- Clarifying the doubts of the patients and attenders. 
- The nurse team uses it as a platform for discussion patient care issues and making a team decision about patient care.

- Motivation of the ward team ${ }^{7}$

Reshma A, Aisha L, Dale A conducted an ethnographic prospective observational study comparing nurse-led and physician-led rounds in General Medicine ward at the National University Hospital in Singapore. The study was conducted among 57 patients. The objective of the study was to determine the average time spend with patients at the bedside for a nurse-led rounds and physicianled rounds. The result suggested that quality time spent with patients at the bedside during morning rounds may be improved by nurse-led rounds when compared to physician led ward rounds ${ }^{8}$.

\section{Importance of nurse led ward rounds for neurological patients.}

The neurological patients are at risk to develop complications, because their sensory and motor reflexes are diminished. The neurological patients are wholly or partially dependent on the nurse. The patient is difficult to arouse and needs constant stimulation in order to follow a simple command. The patient may respond verbally with one or two words, but will drift back to sleep. So the patient needs constant stimulation ${ }^{24}$. The common problems faced by them are unable to mobilize independently, loss of control over bowel and bladder, Poor oral hygiene linked with the development of aspiration pneumonia due to bacterial colonization, Dry mouth, oral ulcers and stomatitis. Falls are also most common among these patients and hence they require continuous and meticulous care and observation ${ }^{9}$. The physician led ward rounds are very brief and nursing care aspects are often overlooked by them and therefore it is important to have nurse led ward rounds to vulnerable patients of all ages who have neurological disorders. In a nurse led ward rounds, accurate and consistent documentation helps to ensure subtle changes in neuro status which is essential to compare the findings with the previous examinations and it also helps to spot changes and intervene any necessary interventions quickly and appropriately $^{19}$.

\section{Role of Nurse in Nurse Led Wards Rounds}

The key aspects of the nurse's role on nurse led ward rounds can be defined using the acronym.

\section{“ACTION"}

- Advocate;

- Chaperone;

- Transitions;

- Informative;

- Organiser;

- Nurse-centred.

\section{Advocate:}

The nurse will be a resourceful person for neurological patients, family and community groups. She will assist with the provision of health promotion activities, she will provide psychological support for the patients who are conscious and also for the care givers of unconscious patients who are wholly dependent ${ }^{10}$.

\section{Chaperone:}

The role of nurse as chaperone includes preparing and assisting in any invasive and non invasive procedures like lumbar puncture, CT, MRI , neurological examination etc. The nurse will also help the patient in communicating and positioning, giving privacy, minimizing anxiety \& potential embarrassment and respecting their cultural issues ${ }^{25}$.

\section{Transitions:}

As a transitor the nurse functions will include noting any ongoing investigation and communicating to the patient and team, documenting any incomplete investigation, referrals and for patient discharge.

\section{Informative:}

The nurse plays a vital role as an informative seeker and giver. The function includes.

Communicate effectively and regularly with patients, parents, and families of wholly and partially dependent patients about the medical status and establish therapeutic relationships. The nurse will also provide appropriate information about the progress, major procedures, prognosis and clinical decision to the patients/family members ${ }^{26}$. The nurse also Communicates effectively with the health care providers including neurology nurses, ward nurses, consulting physicians, residents, medical and nursing students and discuss appropriate information ${ }^{11}$. 


\section{Organizer:}

The role of a nurse as an organizer encompasses, to be informed of the management plans proposed by the referring physician or other consultants for every inpatient to effectively coordinate the neurological aspects of the patients care. The nurse will Contribute effectively in discharge planning for every inpatient with emphasis on the neurological aspects of the patients' ongoing care (e.g. long-term care facility, homecare, parent education, rehabilitation of neurological patients). The nurse will discuss the Discharge planning with the referring physician and other consultants ${ }^{12}$.

\section{Nurse centered:}

The nurse centered role includes Utilizing resources effectively to balance patient care, learning needs, allocating finite health care resources wisely. The nurse will provide appropriate judgment in triaging patients for access to resources which are limited and also establish routines for carrying out regular activities and adhere to them. The role also extends in maintaining complete and accurate medical records. In a nutshell the nurse in-charge of a ward or unit who is clinically overseeing all areas of the ward will provide feedback to the staff nurses and other multidisciplinary team after the ward rounds ${ }^{13}$.

Catangui EJ conducted a study in imperial college health care trust among 108 stroke patients on nurse led ward rounds a valuable contribution to acute stroke care. The objective of the study was to evaluate the stroke related outcomes by initiating nurse led ward rounds. The study concluded that the initiative has been successful in improving clinical communication between nurses and patient involvement in their care planning. It has also empowered nurses to make decisions within their professional arena, and its contribution had an impact on patient care and safety through early detection and prevention of stroke complications. The authors have designed a profoma for nurse led ward rounds to assess the outcomes of stroke patients. It is also used to evaluate whether stoke patients are improving through the provision of nursing care and treatment. The proforma for nurse led ward rounds for stroke patients, includes various dimensions and caring aspect from which the progress of the patient can be identified provide early nursing intervention ${ }^{14}$.

\section{Conclusion:}

A nurse-led ward round has addressed nursing issues in a timely proactive fashion. The initiative has been successful in improving clinical communication between nurses and patient involvement in their care planning. It has enabled the nurses to make decisions within their professional arena, and its contribution has had an impact on patient care and safety through early detection and prevention of neurological complications. ${ }^{15}$

\section{Reference:}

1. Stickrath C, Noble M, Prochazka A, Anderson M, Griffiths M, Manhein J, Sillau S, Aagaard E. Attending rounds in the current era: what is and is not happening. JAMA Intern Med. 2013;173:1084-1989.

2. Weber H, Stockli M, Nubling M, Langewitz WA. Communication during ward rounds in Internal Medicine: an analysis of patient-nurse-physician interactions using RIAS. Patient Educ Couns. 2007;67:343-348.

3. Cohn A. Restore the prominence of the medical ward round. BMJ. 2013;347:f6451.

4. Lees L. The nurse's role in hospital ward rounds. The Nursing Times. 2013.109:12,12-14.

5. Coleman, S., Henneman, E.A. Comprehensive patient care and documentation through unit-based nursing rounds. Clin Nurse Spec. 1991;5:117-120.

6. Catangui EJ, Slark J. Nurse-led ward rounds: a valuable contribution to acute stroke care. Br J Nurs. 2012 Jul 1225;21(13):801-5.

7. Aitken, L.M., Burmeister, E., Clayton, S., Dalais, C., and Gardner, G. The impact of nursing rounds on the practice environment and nurse satisfaction in intensive care: pre-test post-test comparative study. International Journal of Nursing Studies. 2011; 48

8. Reshma A, Aisha L, Dale A. A pilot study on nurse-led rounds: Preliminary data on patient contact. International Journal of Technical Research and Applications.2014. Nov, PP. 68-71.

9. Katherine DS. APN-led nursing rounds: An emphasis on evidence-based nursing care. Journal of the British Association of Critical Care Nurses .2013.May.

10. Bourgault, A.M., King, M.M., Hart, P., Campbell, M.J., Swartz, S., and Lou, M. Circle of excellence. Does regular rounding by nursing associates boost patient satisfaction?. Nursing Management. 2008; 39: 18-24

11. Davies, K.E. Hourly patient rounding. An effective program can decrease call bell usage and patient falls, and increase patient and staff satisfaction. ; 2010 (Advance healthcare network for nurses. Retrieved August 17, 2010.

12. Kalman, M., Olrich, T., and Nigolian, C. Hourly nursing rounds: shaping nursing culture to achieve nursing quality outcomes. Clinical Nurse Specialist. 2008; 22

13. Meade, C.M., Bursell, A.L., and Ketelsen, L. Effects of nursing rounds: on patients' call light use, satisfaction, and safety. American Journal of Nursing. 2006; 106: 5870 (Retrieved September, 2006 ). 
21. Hickey, J. V. (2003). The clinical practice of neurological and neurosurgical nursing (5th ed). Philadelphia: Lippincott

22. Bader, M. K., \& Littlejohns, L. R. (2004). AANN core curriculum for neuroscience nursing (4th ed.). Philadelphia: Saunders.

23. Messner, R., \& Wolfe, S. (1997). RN's pocket assessment guide. Montvale, NJ: Medical Economics.

24. Kerr, M. E. (2000). Intracranial problems. In S. M. Lewis, M. M. Heitkemper, \& S. R. Dirksen (Eds.), Medical surgical nursing (5th ed). St. Louis: Mosby.

25. Neville, K., Lake, K., LeMunyon, D., Paul, D., and Whitmore, K. Nurses' perceptions of patient rounding. Journal of Nursing Administration. 2012; 42: 83-88.

26. Sobaski, T., Abraham, M., Fillmore, R., McFall, D.E., and Davidhizar, R. The effect of routine rounding by nursing staff on patient satisfaction on a cardiac telemetry unit. Health Care Management (Frederick). 2008; 27: 332-

27. Woodard, J.L. Effects of rounding on patient satisfaction and patient safety on a medical-surgical unit. Clinical Nurse Specialist. 2009; 23: 200-206 (Retrieved August, 2009 